\title{
Research on Multi-punch Incremental Sheet Metal Bending Process
}

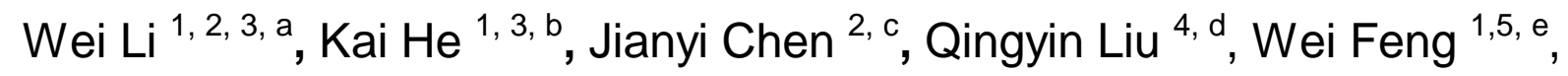 \\ Ruxu Du ${ }^{5, f}$ \\ ${ }^{1}$ Shenzhen Institutes of Advanced Technology, Chinese Academy of Sciences, Shenzhen, China \\ ${ }^{2}$ China University of Petroleum, Beijing, China \\ ${ }^{3}$ Shenzhen Key Laboratory of Precision Engineering, Shenzhen, China \\ ${ }^{4}$ Taian Hualu Metalforming Machine Tool Co., Ltd., Taian, China \\ ${ }^{5}$ The Chinese University of Hong Kong, Hong Kong, China

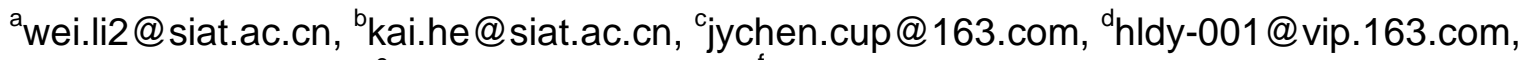 \\ ewei.feng@siat.ac.cn, rrdu@mae.cuhk.edu.hk
}

Keywords: Incremental Forming; Multi-punch; Finite Element; Experimental Analysis.

Abstract. In the process of incremental sheet metal forming, the number of punches has a significant impact on the machining results. Compared with the single-punch forming processing technology, multi-punch processing technology is combined with the advantages of better forming efficiency and higher forming precision. In this paper, 3D finite element model of the incremental forming process is established to calculate the stress and deformation of plates under different processing conditions. In addition, contrast experiments between single-punch forming process and multi-punch forming process have been carried out to compare the surface quality and forming precision of formed plates with the help of 3D optical scanner.

\section{Introduction}

Incremental sheet metal forming technology adopts the idea of rapid prototyping and layered manufacturing technology [1]. The incremental sheet plates forming process has a variety of advantages such as cleaner working environment, higher forming efficiency and better economic benefits, which all make contributions to expanding its application prospects.

The study on the effects of forming parameters arouse the interest of many scholars. Xu Ziran carried out an investigation of the formability of incremental sheet forming under different shapes [2]. Li M studied on the tool-path generation for sheet metal incremental forming based on STL model with defects [3]. The influences of forming tools' size on equivalent plastic strain, forming precision and thickness reduction ratio of deformed parts were studied by Cai Gaipin based on 3D finite element analysis model [4]. Li Jiuhua proposed a new kind of incremental bending method for complicated curved sheet metal [5].

However, all the aforementioned researches hardly focus on the effects of the punch number on the forming performance in the incremental forming process. In this paper, an experimental prototype for incremental sheet metal bending has been designed and manufactured. 3D finite elemental model has been established to simulate the stress and deformation of formed plates after being stamped once and a series of comparative experiments which focus on the forming performance comparison between single punch and three punches have been carried out to validate the simulation results.

\section{Experimental Prototype}

The experimental prototype shown in Fig 1 consists of machine frame, hydraulic supporting platform and the punch group. The punch group includes three punches of the same size, the distances between which can be adjusted according to the forming demands. The hydraulic supporting platform with twenty electromagnetic chucks which are equipped with the function of adsorption plays an important role during the incremental forming process. 3D optical scanner located on the top of the frame is 
responsible for scanning the formed plates and outputting 3D point-cloud data for further processing, which provides measuring means for comparison experiments.
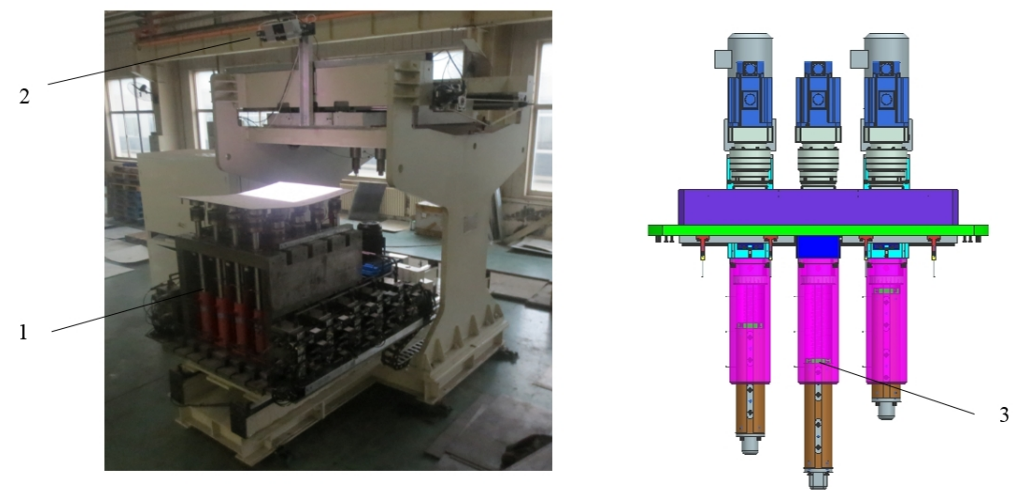

Fig 1. Mechanical structure of the machine

1.Hydraulic supporting platform 2.Optical sacnner 3. Punch group

The bending process is incrementally implemented by the punch group with single punch or all the three punches according to specified processing parameters. During the forming process, the formed plate is supported by hydraulic support columns with the help of electromagnetic chucks and the hydraulic columns are designed to move in accord with the processing path. In this way, the curved plate will be achieved step by step until its complex shape gets consistent with the design model.

The processing path of incremental sheet plate forming which is generated based on the symmetric principle and minimum path principle is shown in Fig 2. The circles with grey shades represent the position of twenty magnetic chucks on the hydraulic support platform. The circles with numbers which express the forming sequence are supposed to show the stamping position of the punch group. The layer number of incremental forming is determined based on the given design model and the forming depth of punch group increases layer by layer. The machining time of three-punch forming process is supposed to be much shorter due to its higher forming efficiency.

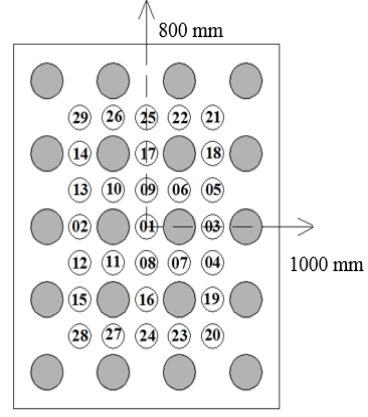

(a) Single Punch

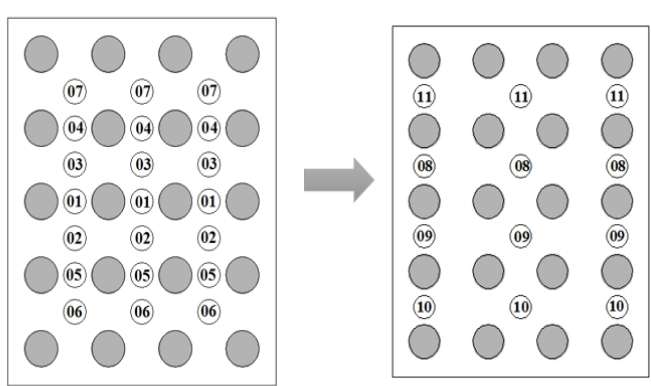

(b) Three Punches

Fig 2. Processing path of incremental plate forming

\section{Finite Element Analysis}

In this paper, LS_DYNA, focusing on analyzing the characteristics of two different forming processes, is used to calculate the stress and deformation of formed plates after being stamped once. The diameter of punch is $50 \mathrm{~mm}$ and the processing depth of each punch is also $50 \mathrm{~mm}$. The coordinate of the action spot in single-punch simulation is $(200,0)$ and the coordinates of the action spots in three-punch simulation are $(-200,0),(0,0)$ and $(200,0)$.

The stress nephograms of formed plates after stamping are shown in Fig 3. In the single-punch forming process, the action spot is not in the center of the plate, resulting in the uniform stress distribution on the plate. However, the stress distribution in the three-punch forming process is symmetrical along the central line accompanied by a reduction from the middle to the edge. In the single-punch forming process, deformation of plate on the line of $y=0$ increases gradually along the 
$\mathrm{X}$-axis and the deformation on the line of $\mathrm{y}=0$ in three-punch forming process is consistent and the strain curve is almost a straight line.
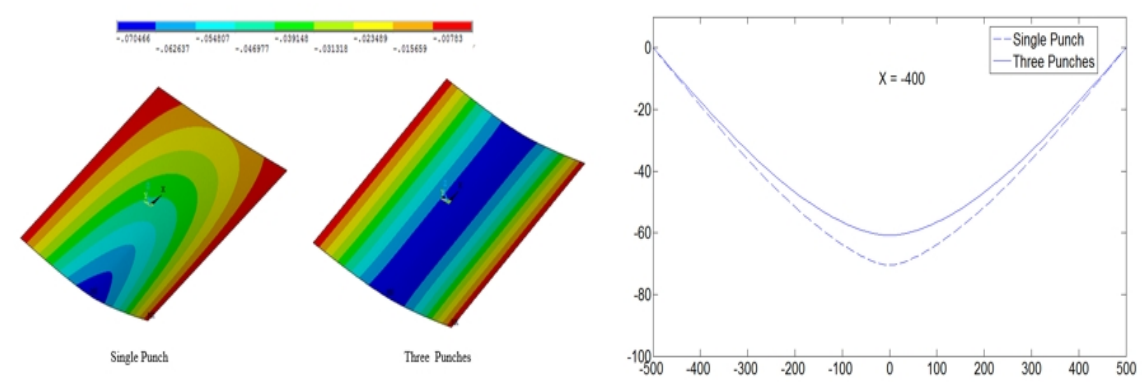

Fig 3. Stress and Deformation

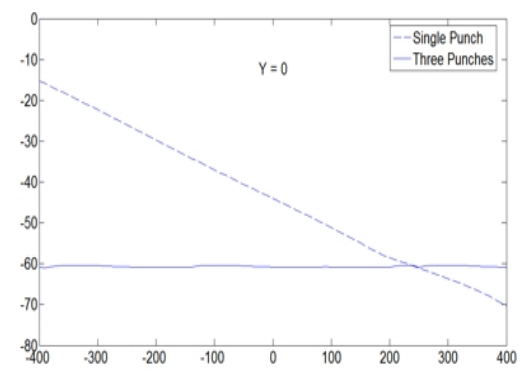

Through the simulation calculation, it can be concluded that the stress and strain distribution of formed plates in three-punch forming process is more uniform compared with that in single-punch forming process. The progressive deformation of formed plate in three-punch forming process is well-distribution, guaranteeing higher precision of incremental plate bending process. In the following section, contrast experiments will be carried out to validate the simulation results.

\section{Experimental Analysis}

In this section, plates will be incrementally formed by single punch and three punches according the same design model to compare the processing effects between single-punch forming process and three-punch forming process.

The design model with single curvature is shown in Fig 4(a) and its maximum depth along the Z-axis is $70 \mathrm{~mm}$. As shown in Fig 4(b), the depth of design model with gradual curvature increases from the position at $\mathrm{x}=-400$ to the position at $\mathrm{x}=400$ along the line of $\mathrm{y}=0$. The formed plates whose surfaces were sprayed with developer in order to make it convenient for 3D optical scanner to work are shown in Fig 5.

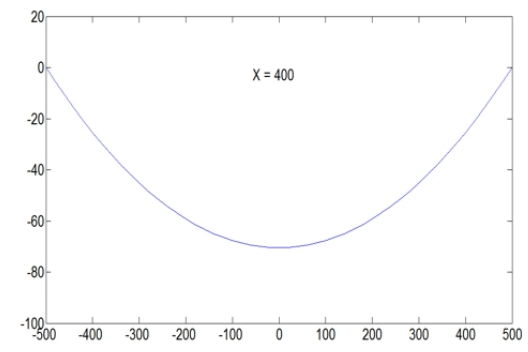

(a)

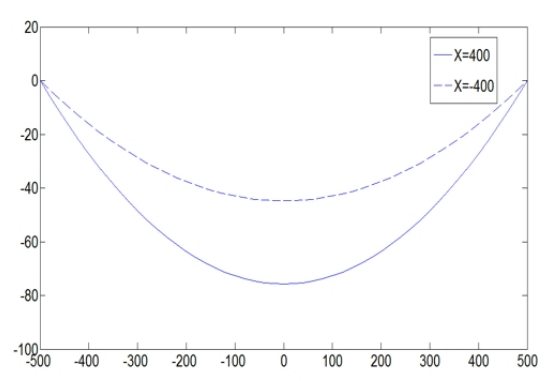

(b)

Fig 4. Design models

a.Design model with single curvature $b$. Design model with gradual curvature

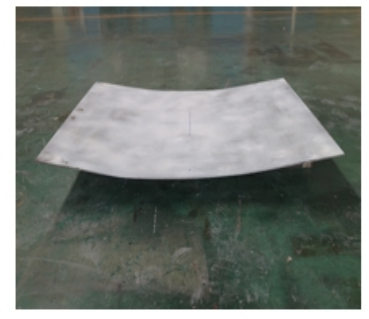

(a)

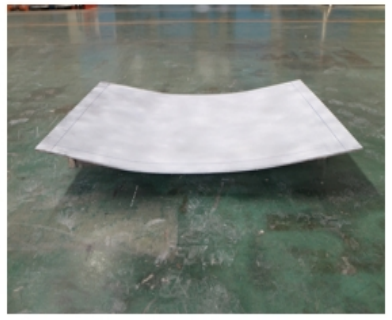

(b)

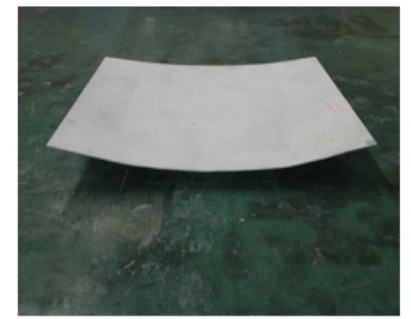

(c)

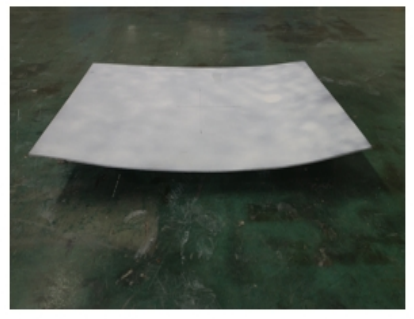

(d)

Fig 5. Formed plates

a.Single curvature (single punch) b.Single curvature (three punches) c. Grdual curvature (single punch) d.Grdual curvature (three punches) 
After the incremental forming process was completed, point-cloud data of the formed plates was acquired with the help of 3D optical scanner for further processing and strain curves of formed plates were outputted for comparative analysis.

The profile curves of formed plates generated by MATLAB software according to the coordinate data are shown in Fig 6 . The profiles of shaped plates are basically in accord with the design model, but the maximum depth of each shaped plate is smaller than that of the design model because of rebound effect. Compared with single-punch forming process, three-punch forming process has the advantage of higher forming accuracy and better symmetry with smaller springback. Due to optimized processing path, three-punch forming process delivers time saving of 30\% compared to single-punch forming process.
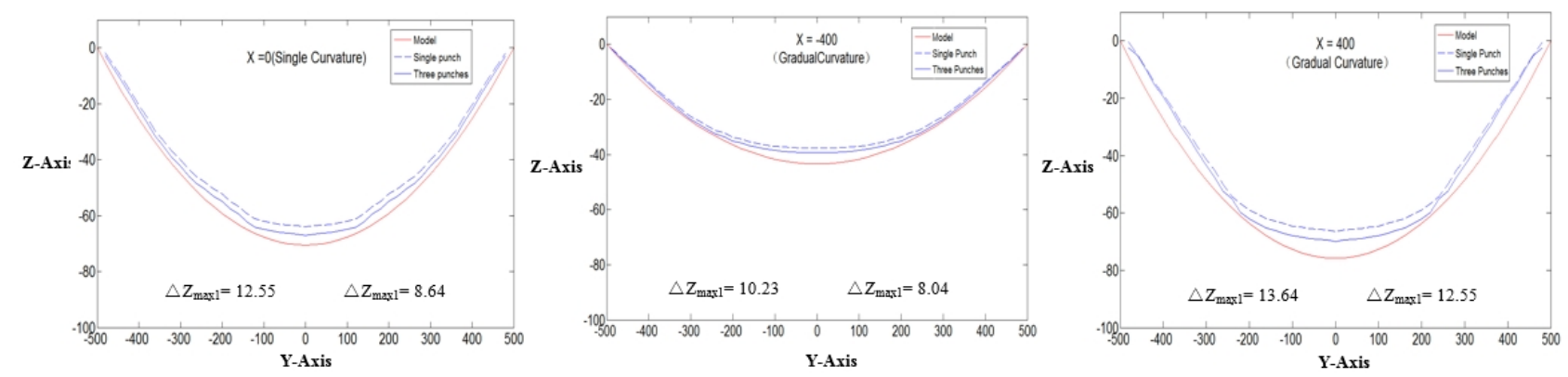

Fig 6. Experimental comparison of formed plates

\section{Summary}

In this paper, simulation analysis and experiments focusing on the incremental sheet plate forming have been carried out to figure out the processing characteristics of multi-punch forming process compared to single-punch forming process under the same machining parameters. The results indicate that multi-punch forming process has the characteristics of higher forming accuracy and better forming efficiency due to uniform stress and strain distribution and optimized processing path.

\section{Acknowledgements}

This research is supported by Taishan Scholar of Leading Talent in Ocean Engineering - A Special Project of Numerical Control Ship Building Machines, Shenzhen Basic Research Project under the grant number JCYJ20150521094519483 and Key Cooperative Program of the Bureau of International Co-operation Chinese Academy of Sciences (No.172644KYSB20160024).

\section{References}

[1] Sena J I V, Alves de Sousal R J, Valente R A F. Single point incremental forming simulation with an enhanced assumed strain solid-shell finite element formulation. Int. J. Form. , 2010, 3 (1): 963 966.

[2] $\mathrm{Xu} \mathrm{Z} \mathrm{R,} \mathrm{Gao} \mathrm{L,} \mathrm{Cui} \mathrm{Z,} \mathrm{et} \mathrm{al.} \mathrm{Investigation} \mathrm{of} \mathrm{the} \mathrm{formability} \mathrm{of} \mathrm{incremental} \mathrm{sheet} \mathrm{forming} \mathrm{under}$ different shapes. Mechanical Science and Technology for Aerospace Engineering, 2009, 28 (5): 614 617.

[3] Li M, Zhang L C, Mo J H, et al. Tool-path generation for sheet metal incremental forming based on STL model with defects. Int. J. Adv. Manuf. Technol., 2012, 63: 535 - 547.

[4] Jiuhua Li, Xiaobing Dang, Kai He, et al. A new kind of incremental bending method for complicated curved sheet mental. International Multi-Conference on Engineer and Technology Innovation 2015, Kaohsiung, Taiwan, October 30-November 032015.

[5] Dang X B, He K, Wei S H, et al. Application of Incremental Forming in Sheet Metal Bending Process. Advanced Materials Research, 2014, 99: 561 -564. 